\title{
Relaxation Time Coupling Method for Ultrasonic Sensor Design
}

\author{
Hu Yi, Wang Shu, Zhu Ming \\ School of Electronic Information and Communications, Huazhong University of Science and Technology, Wuhan 430074, China
}

\begin{abstract}
In our previous work [Sens. Actuator B: Chem. 203 1-8 (2014)], a ultrasonic sensor has been proposed to detect gas compositions by the acoustic spectral peak location. However, the effective relaxation area constructed by existing theoretical model cannot match the detection method when there are more than one strong relaxational components in gas mixture. A method that calculates the relaxation time of a multi-component relaxation process is presented. Based on acquirement of decoupled relaxation times and their corresponding effective specific heat values, it calculates the whole relaxation time of a multi-component relaxation process. The method is based on the fact that for a multi-component gas mixture, such as occurs in many polyatomic gas mixtures, the relaxation process should be considered as a whole rather than being decoupled as some single relaxation processes. Acquiring relaxation time provides the approach to obtain the acoustic absorption spectral peak value directly.
\end{abstract}

\section{Introduction}

Intelligent ultrasonic sensor is applied widely to detect gas compositions [1]. Measuring acoustic relaxation absorption spectrum is a powerful method to characterize molecular relaxation process in gas mixture $[1,2,3]$. For the complexity of obtaining the whole spectrum, we proposed a method to employ the spectral peak location to detect gas composition. Based on this method, a gas detection sensor with two pairs of ultrasonic transducers are suggested to practical applications [4]. In a simple relaxation process, the acoustic relaxation absorption spectral peak location is determined by the relaxation time $[5,6]$. Therefore, calculating the relaxation time in excitable gases is a key issue to apply the spectral peak location based method in gas detection.

Over the years, some relaxation time theories have been proposed [7-10]. However, these theories did not provide an appropriate physical model to demonstrate the theoretical derivation. Recently, based on the decoupling model in multi-component excitable gases [11], Zhang calculated the vibrational relaxation times of the specific decoupled relaxation processes [6]. However, a multicomponent relaxation process should be considered as a whole physical process. Zhang's model can only calculate the decoupled relaxation times of every single relaxation processes. In practical detection, calculating the acoustic absorption spectral peak focuses on the whole relaxation time of a multi-component relaxation process.

Based on the relaxation time theories, we develop a model in this paper to calculate the whole relaxation time. The model can be applied to measure the spectral peak value, i.e. the effective relaxation frequency and the maximum acoustic absorption coefficient. In the paper, we propose a relaxation time coupling based method to obtain the whole relaxation time. Then we compare the predicted results based on the proposed method with the experimental data, and provide the experimental results in strong-strong relaxational gas mixtures.

\section{Coupling relaxation times method}

The acoustic relaxation absorption decoupling model [11] suggests that a multi-component vibrational relaxation process in excitable gases can be decoupled to some single relaxation processes. Effective isochoric specific heat $C_{V}^{\text {eff }}$ of multi-component relaxation process can be characterized by the relaxation times as $[6,11]$ :

$$
C_{V}^{\mathrm{eff}}=C_{V}^{\infty}+\sum_{n=1}^{N} \frac{C_{n}^{*}}{1+i \omega \tau_{n}}
$$

where $C_{V}^{\text {eff }}$ represents the macroscopic 'footprint' of the inability of the internal degrees of freedom to follow the external temperature fluctuations [12]. $C_{V}^{\infty}$ is the highfrequency instantaneous limit value, $C_{V}^{\infty} \equiv \lim _{\omega \rightarrow \infty} C_{V}^{\text {eff }}(\omega)$. The $C_{V}^{\infty}$ is only dependent on the molecular symmetry. For a linear molecule, $C_{V}^{\infty}=5 \mathrm{R} / 2$, and for a nonlinear molecule, $C_{V}^{\infty}=3 \mathrm{R}$, $\mathrm{R}$ is universal gas constant. There is another lowfrequency limit $C_{V}^{0} \equiv \lim _{\omega \rightarrow 0} C_{V}^{\mathrm{eff}}(\omega)$. Therefore, the 
difference between the two limiting values represents the contribution of the relaxing internal degrees of freedom of the molecules, $C^{\text {int }}=C_{V}^{0}-C_{V}^{\infty}=C_{V}^{\text {vib }}[5] . C_{n}^{*}$ and $\tau_{n}$ are the isochoric molar specific heat and the relaxation time for one of the decoupled single-relaxation processes, respectively.

Viewed from the gas vibration modes, we can write the $C_{V}^{\text {eff }}$ as [3]:

$$
C_{V}^{\mathrm{eff}}=C_{V}^{\infty}+\sum_{j=1}^{N} a_{j} C_{j}^{\mathrm{vib}} y_{j}, y_{j}=\mathrm{d} T_{j}^{\mathrm{vib}} / \mathrm{d} T
$$

where $y_{j}$ is the ratio between the temperature fluctuation of vibratioanl mode $j\left(\mathrm{~d} T_{j}^{\mathrm{vib}}\right)$ and that of the external DOF ( $\mathrm{d} T$ ). For a gas mixture with $N$ kinds of vibrational modes, $C_{V}^{\mathrm{vib}}=\sum_{j=1}^{N} a_{j} C_{j}^{\mathrm{vib}}, a_{j}$ is the mole fraction of mode $j, C_{j}^{\mathrm{vib}}$ is the vibrational isochoric molar specific heat of mode $j$. The $C_{j}^{\mathrm{vib}}$ is given by the Planck-Einstein function for a harmonic oscillator [2].

Based on Eq. (2) and the general relaxation equation provided in [11], we can obtain the expression of the algebraic relaxation equation in a matrix form [6]:

$$
(\mathbf{R}+\mathrm{i} \omega \mathbf{I}) \mathbf{y}=\mathbf{H}
$$

where $\quad \mathbf{y}_{j}=y_{j} \quad, \quad \mathbf{R}_{j j}=k_{j j}, \quad \mathbf{R}_{j k}=-k_{j k}$, $\mathbf{H}_{j}=k_{j j}-\sum_{\substack{k=1 \\ k \neq j}}^{N} k_{j k}, \mathbf{I}$ is the identity matrix. Ref. [11] defined the matrix $\mathbf{R}$ as energy transition rate matrix. We can construct the matrix $\mathbf{R}$ by calculating the $k_{j j}$ and $k_{j k}$ as [6]:

$$
\begin{gathered}
k_{j j}=k_{10}(j)+\sum_{\substack{k=1 \\
k \neq j}}^{N} k_{01}^{10}(j, k) \frac{1-\exp \left(-h v_{j} / k_{\mathrm{B}} T_{0}\right)}{1-\exp \left(-h v_{k} / k_{\mathrm{B}} T_{0}\right)}, \\
k_{j k}=k_{01}^{10}(j, k) \frac{v_{k}}{v_{j}} \frac{1-\exp \left(-h v_{j} / k_{\mathrm{B}} T_{0}\right)}{1-\exp \left(-h v_{k} / k_{\mathrm{B}} T_{0}\right)},
\end{gathered}
$$

where $v_{j}$ and $v_{k}$ are the vibrational frequency of mode $j$ and $k$, respectively; $h$ is Planck's constant; $k_{\mathrm{B}}$ is Boltzmann's constant; $T_{0}$ is the equilibrium temperature. $k_{10}(j)$ and $k_{01}^{10}(j, k)$ are the $\mathrm{V}-\mathrm{T}$ transition rate and the $\mathrm{V}-\mathrm{V}$ transition rate respectively. They can be calculated by the Tanczos equation $[1,3]$. By using Eq. (1) and Eq. (3), we can obtain the relaxation times $\tau_{n}$ and the isochoric molar specific heat $C_{n}^{*}$ of the decoupled singlerelaxation processes. The reciprocals of the eigenvalues of the energy transition rate matrix $\mathbf{R}$ are exactly the relaxation times $\tau_{n}$ [6]. The detailed derivation is developed in [6].

For a multi-component relaxation process, refs. [6] and [11] decoupled the whole relaxation process to some single relaxation processes, and allocated the relaxation times to every specific relaxation process as Eq. (1). Based on the decoupling model, Zhang et al. suggested that there will be as many relaxation times as there are vibrational modes available to a gas, and the relaxation time of the primary relaxation process determines the spectral peak location in most cases [6]. However, we think that the "primary relaxation time" in [6] is actually the first relaxation time calculated based on the decoupling model. If there are two strong relaxational gases in mixture, $C_{1}^{*}$ and $C_{2}^{*}$ are in the same order of magnitude, meanwhile the first relaxation time $\tau_{1}$ of $C_{1}^{*}$ and the second relaxation time $\tau_{2}$ of $C_{2}^{*}$ in Eq. (6) are approximate. In this case, the whole relaxation time can not obtained by the decoupling model. Consequently, we proposes a coupling relaxation times method to obtain the whole relaxation time of a multimode relaxation process.

Actually, we consider the effective isochoric specific heat $C_{V}^{\text {eff }}$ of a multi-component relaxation process can be given as [5]:

$$
C_{V}^{\mathrm{eff}}=C_{V}^{\infty}+\frac{C^{\mathrm{int}}}{1+i \omega \tau_{\mathrm{m}}}
$$

where $\tau_{\mathrm{m}}$ is $C^{\text {int }}$ is the difference between the $C_{V}^{0}$ and $C_{V}^{\infty}$, represents the contribution of the relaxing internal degree of freedom of the molecules, $\tau_{\mathrm{m}}$ is the whole relaxation time of the whole relaxation process [5].

Since there are no more than two significant relaxation processes appearing together in the available literatures, we focus on the case that $C_{1}^{*}$ and $C_{2}^{*}$ are both significant. Therefore we can rewrite the Eq. (1) as:

$$
C_{V}^{\mathrm{eff}}=C_{V}^{\infty}+\frac{C_{1}^{*}}{1+i \omega \tau_{1}}+\frac{C_{2}^{*}}{1+i \omega \tau_{2}}
$$

where $C_{1}^{*}$ and $\tau_{1}$ are the isochoric molar specific heat and the relaxation time of the first significant relaxation process; $C_{2}^{*}$ and $\tau_{2}$ are the isochoric molar specific heat and the relaxation time of the second significant relaxation process. Combining Eq. (5) and (6), the equation can be obtained:

$$
\frac{C^{\mathrm{int}}-i \omega \tau_{\mathrm{m}} C^{\mathrm{int}}}{1+\omega^{2} \tau_{\mathrm{m}}{ }^{2}}=\frac{C_{1}^{*}-i \omega \tau_{1} C_{1}^{*}}{1+\omega^{2} \tau_{1}^{2}}+\frac{C_{2}^{*}-i \omega \tau_{2} C_{2}^{*}}{1+\omega^{2} \tau_{2}^{2}}
$$


To the two strong relaxation components gas mixtures, $\tau_{1} \approx \tau_{2}$, thus the right hand side of the equation is $\frac{C_{1}^{*}+C_{2}^{*}-i \omega\left(\tau_{1} C_{1}^{*}+\tau_{2} C_{2}^{*}\right)}{1+\omega \tau_{1} \tau_{2}}$. In Eq. (7), since $\tau_{1} \approx \tau_{2}$, $C_{1}^{*}$ and $C_{2}^{*}$ are much large than the other $C_{n}^{*}$, we have $C^{\text {int }} \approx C_{1}^{*}+C_{2}^{*}$. Based on this approximation, we can conclude that $1+\omega^{2} \tau_{\mathrm{m}}{ }^{2}=1+\omega \tau_{1} \tau_{2}$. Then from Eq. (7), $\tau_{\mathrm{m}}$ is given by:

$$
\tau_{\mathrm{m}}=\frac{C_{1}^{*}}{C_{1}^{*}+C_{2}^{*}} \tau_{1}+\frac{C_{2}^{*}}{C_{1}^{*}+C_{2}^{*}} \tau_{2}
$$

$C_{n}^{*} / \sum C_{n}^{*}$ can be considered as its contribution to the total vibrational heat capacity, the relaxation time $\tau_{\mathrm{m}}$ is a linear combination of the decoupled relaxation times with the weighted factor $C_{n}^{*} / \sum C_{n}^{*}$ :

$$
\tau_{\mathrm{m}}=\sum_{n}^{N} \frac{C_{n}^{*}}{\sum C_{n}^{*}} \tau_{n}
$$

With the relaxation time $\tau_{\mathrm{m}}$, acoustic relaxation absorption spectra $\mu_{\mathrm{r}}(\omega)$ can be expressed as $[13,14]$ :

$$
\mu_{\mathrm{r}}(\omega)=\pi \tau_{\mathrm{m}} \omega \mathrm{R} \frac{C^{\text {int }}}{\left(\mathrm{R}+C_{V}^{0}\right) C_{V}^{0}+\omega^{2} \tau_{\mathrm{m}}^{2}\left(\mathrm{R}+C_{V}^{\infty}\right) C_{V}^{\infty}}
$$

where $\omega=2 \pi f$ is the acoustic angular frequency, $\mu_{\mathrm{r}}(\omega)$ is the frequency dependence dimensionless acoustic relaxation absorption spectrum, $\mathrm{R}$ is universal gas constant. Based on Eq. (10), we can obtain the relaxation frequency and the maximal acoustic relaxation absorption coefficient, i.e. the acoustic relaxation absorption spectral peak value are:

$$
\begin{gathered}
f_{\text {relax }}=\frac{1}{2 \pi \tau}\left[\left(\mathrm{R}+C_{V}^{0}\right) C_{V}^{0} /\left(\mathrm{R}+C_{V}^{\infty}\right) C_{V}^{\infty}\right]^{1 / 2}, \\
\mu_{\mathrm{m}}=\mu_{\mathrm{r}}\left(2 \pi f_{\text {relax }}\right)
\end{gathered}
$$

\section{Simulation results and discussion}

Based on Eq. (11), we will validate the proposed method by comparing the spectral peak predicted by our method with the experimental data. We first consider the simulation in the mixture $\mathrm{CO}_{2}-\mathrm{N}_{2}$ and $\mathrm{CH}_{4}-\mathrm{N}_{2}$. They both have only one single strong relaxation process. The results are shown in Fig. 1.

As $60 \% \mathrm{CO}_{2}-40 \% \mathrm{~N}_{2}$ shown in Fig. 1(a), the curve represents the acoustic absorption spectrum predicted by the model given in [6], the diamond is its predicted peak.
Then we select five pairs of the experimental data given by Ejakov et al. [15], they are given as the Plused-Circle in Fig. 1, and the other circles are also experimental data. With these five pairs of data, we synthesize them to a measured spectral peak by the algorithm proposed in [5]. The synthesized results are shown as Plus in Fig. 1(a). Finally, we calculate the peak value by our proposed method, the result is shown as Bold Cross in Fig. 1(a).

In Fig. 1(a), the spectral peak predicted by our proposed method (Bold Cross) is closer to the synthesized results (Plus) than the prediction in [6] (Diamond). Similarly, for the $60 \% \mathrm{CH}_{4}-40 \% \mathrm{~N}_{2}$ mixture, the spectral peak predicted by our proposed method is also closer to the synthesized results. The numerical values and relative errors are given in Table 1.

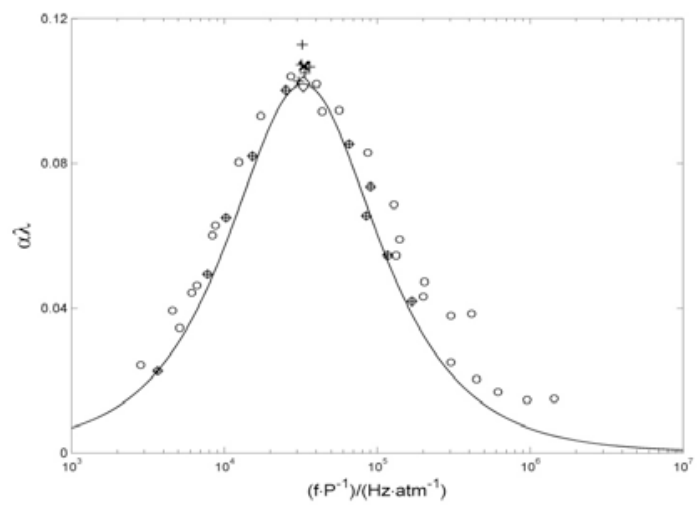

(a)

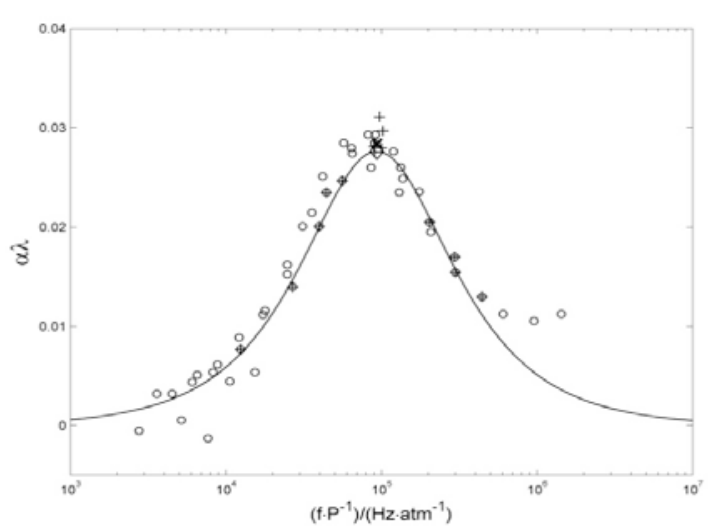

(b)

Figure 1. Comparisons of the synthesized results with the spectral peaks predicted by our method and the analytical model in [6]. a: mixture of $60 \% \mathrm{CO}_{2}-40 \% \mathrm{~N}_{2}$ at $T=293.5 \mathrm{~K}$; $\mathbf{b}$ : mixture of $60 \% \mathrm{CH}_{4}-40 \% \mathrm{~N}_{2}$ at $T=293.4 \mathrm{~K}$.

According to Table 1, for the $60 \% \mathrm{CO}_{2}-40 \% \mathrm{~N}_{2}$ mixture, predicting by our proposed method, the maximum relative error is $5.2732 \%$, and the average relative error is $1.9367 \%$. Yet for the spectral peak predicted by the analytical model in [6], the maximum relative error is $8.2840 \%$, and the average relative error is $3.2347 \%$. Similarly, for the $\mathrm{CH}_{4}-\mathrm{N}_{2}$ mixture, the maximum relative error and average relative error between the prediction results by our proposed method and the measurements are much smaller than the analytical model in [6]. Consequently, it is obvious that 
our proposed method can obtain the relaxation time more correctly than the model in [6].

Table 1. Calculation results of the spectral peaks in Fig. 1 for the mixtures of $60 \% \mathrm{CO}_{2}-40 \% \mathrm{~N}_{2}$ at $T=293.5 \mathrm{~K}$ and $60 \% \mathrm{CH}_{4}$ $40 \% \mathrm{~N}_{2}$ at $T=293.4 \mathrm{~K}$. $\left(3.232^{4}, 0.1098\right)$ means the values of $x$ axis acoustic frequency $(\mathrm{Hz})$ with $1 \mathrm{~atm}$ and $y$-axis

dimensionless acoustic absorption coefficients $\mu_{\mathrm{r}}(\omega)$ of the spectral peak, and the notation $x^{y}$ implies $x \times 10^{y}$.

\begin{tabular}{|c|c|c|c|c|c|}
\hline \multirow[b]{2}{*}{$\begin{array}{c}\text { Mixture } \\
\quad \mathrm{s}\end{array}$} & \multirow{2}{*}{$\begin{array}{c}\text { Synthesize } \\
\text { d result } \\
\text { (Plus) }\end{array}$} & \multirow[b]{2}{*}{$\begin{array}{c}\text { Our } \\
\text { predictio } \\
\text { ns (Bold } \\
\text { cross) }\end{array}$} & \multirow{2}{*}{\multicolumn{2}{|c|}{$\begin{array}{l}\text { Model in } \\
{[6]} \\
\text { predictio } \\
\text { ns } \\
\text { (Diamon } \\
\text { d) }\end{array}$}} & \multirow[b]{2}{*}{$\begin{array}{l}\text { Relati } \\
\text { e error }\end{array}$} \\
\hline & & & & & \\
\hline \multirow{10}{*}{$\begin{array}{l}60 \% \mathrm{CO}_{2} \\
-40 \% \mathrm{~N}_{2}\end{array}$} & $\left(3.232^{4}\right.$ & & 5.2732 & & 8.2840 \\
\hline & $0.1098)$ & & $\%$ & & $\%$ \\
\hline & $\left(3.188^{4}\right.$ & & 0.4794 & & 3.3531 \\
\hline & $0.1048)$ & & $\%$ & & $\%$ \\
\hline & $\left(3.095^{4}\right.$, & $\left(3.265^{4}\right.$, & 0.0959 & $\left(3.285^{4}\right.$ & 2.7613 \\
\hline & $0.1042)$ & $0.1043)$ & $\%$ & $0.1014)$ & $\%$ \\
\hline & $\left(2.752^{4}\right.$ & & 1.1505 & & 1.6765 \\
\hline & $0.1031)$ & & $\%$ & & $\%$ \\
\hline & $\left(2.566^{4}\right.$, & & 2.6846 & & 0.0986 \\
\hline & $0.1015)$ & & $\%$ & & $\%$ \\
\hline \multirow{10}{*}{$\begin{array}{l}60 \% \mathrm{CH}_{4} \\
-40 \% \mathrm{~N}_{2}\end{array}$} & $\left(9.697^{4}\right.$, & & 9.5745 & & 13.187 \\
\hline & $0.0309)$ & & $\%$ & & $\%$ \\
\hline & $\left(1.022^{5}\right.$, & & 4.6010 & & 8.0586 \\
\hline & $0.0295)$ & & $\%$ & & $\%$ \\
\hline & $\left(8.931^{4}\right.$, & $\left(9.358^{4}\right.$, & 1.0638 & $\left(9.332^{4}\right.$ & 2.1978 \\
\hline & $0.0279)$ & $0.0282)$ & $\%$ & $0.0273)$ & $\%$ \\
\hline & $\left(9.998^{4}\right.$ & & 1.4184 & & 1.8315 \\
\hline & $0.0278)$ & & $\%$ & & $\%$ \\
\hline & $\left(9.038^{4}\right.$ & & 2.8369 & & 0.3663 \\
\hline & $0.0274)$ & & $\%$ & & $\%$ \\
\hline
\end{tabular}

To validate our proposed method for the mixture with two strong relaxation gases in the mixture, five gas mixtures are considered: $76 \% \mathrm{CH}_{4}-24 \% \mathrm{CO}_{2}, 81 \% \mathrm{CH}_{4}-$ $19 \% \mathrm{CO}_{2}, \quad 86 \% \mathrm{CH}_{4}-14 \% \mathrm{CO}_{2}, \quad 91 \% \mathrm{CH}_{4}-9 \% \mathrm{CO}_{2}$, and $96 \% \mathrm{CH}_{4}-4 \% \mathrm{CO}_{2}$. We calculate the single relaxation times by the model in [6] and the whole relaxation time by our proposed method, respectively. Then with the calculation results, the spectral peaks are predicted. Finally, we compare the predicted spectral peak value with the measurements data by the synthesizing algorithm [5]. The prediction results and relative errors are given in Table. 2 .

As shown in Table. 2, when the concentration of $\mathrm{CO}_{2}$ is $4 \%$, the relative errors are $1.10 \%$ and $1.99 \%$, respectively. This is because that the major component of the gas mixture is $\mathrm{CH}_{4}$, the mixture can be thought as pure $\mathrm{CH}_{4}$ actually. However when the concentration of $\mathrm{CO}_{2}$ is $24 \%$, the relative error between the measured result and the predicted by our proposed method is $5.14 \%$, while the relative error is $17.37 \%$ for the model in [6]. The spectral peak value predicted by our proposed method are more accurate than that predicted by the model in [6].

\section{Conclusion}

In this paper, we improve the relaxation time model [6] to calculate the whole relaxation time of a multicomponent relaxation process. Based on our proposed method, the spectral peak value can be obtained more correctly than the model in [6]. The relative error between the measured results and the predictions can be effectively decreased by the proposed method. Moreover, for most excitable gases, the relaxation time of the multi relaxation process should be considered as a whole rather than the decoupled first relaxation time that proposed in [6]. Consequently, the acoustic absorption spectral peak predicted by our proposed method can be more correctly than that predicted by the previous theory. The proposed coupling relaxation time method provides idea for intelligent ultrasonic sensor design in practical applications. This work is supported by National Natural Science Foundation of China (Grant Nos. 61371139, 61571201 and 61461008).

Table 2. Calculation results of the spectral peaks in Fig. 3 for the mixtures of $\mathrm{CH}_{4}-\mathrm{CO}_{2}$. The notation $x^{y}$ implies $x \times 10^{y}$.

\begin{tabular}{ccccc}
\hline Mixtures & $\begin{array}{c}\text { Synthesized } \\
\text { results }\end{array}$ & $\begin{array}{c}\text { Our } \\
\text { method }\end{array}$ & $\begin{array}{c}\text { Model in } \\
{[6]}\end{array}$ & $\begin{array}{c}\text { Relative } \\
\text { error }\end{array}$ \\
\hline $76 \% \mathrm{CH}_{4^{-}}$ & $\left(1.463^{5}\right.$, & $\left(1.138^{5}\right.$, & $\left(1.464^{5}\right.$, & $5.14 \%$ and \\
$24 \% \mathrm{CO}_{2}$ & $0.05459)$ & $0.05192)$ & $0.04651)$ & $17.37 \%$ \\
$81 \% \mathrm{CH}_{4^{-}}$ & $\left(1.390^{5}\right.$, & $\left(1.118^{5}\right.$, & $\left(1.377^{5}\right.$, & $4.57 \%$ and \\
$19 \% \mathrm{CO}_{2}$ & $0.04970)$ & $0.04753)$ & $0.04363)$ & $13.91 \%$ \\
$86 \% \mathrm{CH}_{4^{-}}$ & $\left(1.396^{5}\right.$, & $\left(1.131^{5}\right.$, & $\left(1.309^{5}\right.$, & $3.08 \%$ and \\
$14 \% \mathrm{CO}_{2}$ & $0.04451)$ & $0.04318)$ & $0.04058)$ & $9.68 \%$ \\
$91 \% \mathrm{CH}_{4^{-}}$ & $\left(1.298^{5}\right.$, & $\left(1.165^{5}\right.$, & $\left(1.271^{5}\right.$, & $1.08 \%$ and \\
$9 \% \mathrm{CO}_{2}$ & $0.03931)$ & $0.03889)$ & $0.03726)$ & $5.50 \%$ \\
$96 \% \mathrm{CH}_{4^{-}}$ & $\left(1.241^{5}\right.$, & $\left(1.209^{5}\right.$, & $\left(1.235^{5}\right.$, & $1.10 \%$ and \\
$4 \% \mathrm{CO}_{2}$ & $0.03426)$ & $0.03464)$ & $0.03359)$ & $1.99 \%$ \\
\hline
\end{tabular}

\section{References}

1. Phillips S, Dain Y, Lueptow R M Meas. Sci. Technol. 1470 (2003)

2. Dain Y, Lueptow R M J. Acoust. Soc. Am. 1091955 (2001)

3. Zhang K S, Wang S, Zhu M, Hu Y, Jia Y Q Acta Phys. Sin. 61174301 (in Chinese) (2012)

4. Hu Y, Wang S, Zhu M, Zhang K S, Liu T T, Xu D Y Sens. Actuator B-Chem. 2031 (2014)

5. Petculescu A G, Lueptow R M Phys. Rev. Lett. 94 238301 (2005)

6. Zhang K S, Ou W H, Jiang X Q J. Korean Phys. Soc. 651028 (2014)

7. Schwartz R N, Slawsky Z I, Herzfeld K F J. Chem. Phys. 201591 (1952) 
8. Tanczos F I J. Chem. Phys. 25439 (1956)

9. Dain Y, Lueptow R M J. Acoust. Soc. Am. 1102974 (2001)

10. Petculescu A G, Lueptow R M J. Acoust. Soc. Am. 117175 (2005)

11. Zhang K S, Wang S, Zhu M, Ding Y, Hu Y Chin. Phys. B 22014305 (2013)

12. Zhang K S, Wang S, Zhu M, Ding Y Meas. Sci. Technol. 24055002 (2013)
13. Herzfeld K F, Litovitz T A Absorption and Dispersion of Ultrasonic Waves (New York: Academic) p55-216 (1959)

14. Lambert J D Vibrational and Rotational Relaxation in Gases (Oxford: Clarendon) p1-114 (1977)

15. Ejakov S G, Phillips S, Dain Y, Lueptow R M, Visser J H J. Acoust. Soc. Am. 1131871 (2003) 